\title{
Sensitivity and Figure of Merit Measurements of EELS vs XEDS in the Analytical Electron Microscope
}

Nestor Zaluzec ${ }^{1}$ and Magnus Garbrecht ${ }^{2}$

${ }^{1}$ Argonne National Laboratory, Bolingbrook, Illinois, United States, ${ }^{2}$ The University of Sydney, Sydney, New South Wales, Australia

The comparison of the relative sensitivity of X-ray Energy Dispersive Spectroscopy (XEDS) versus Electron Energy Loss Spectroscopy (EELS) has been a long-standing discussion/debate in the Analytical Electron Microscopy community [1]. While it is without question that the absolute signal generation of EELS compared to XEDS is greater, measures of the sensitivity that applies equally to both spectroscopies have rarely been quantitatively assessed consistently. In this work, a definition for figure of merit (FoM) based upon the measurements of the variation of Signal \& Signal/Background as a function of thickness has been developed and experimentally tested.

The detectability limit of a characteristic spectroscopic signal has been traditionally defined by the Student $\mathrm{T}$-Test at the $95 \%$ criteria namely that the changed in measured signal $\Delta \mathrm{I}_{\mathrm{M}}$ should be greater than 2.33 times it's variance [2]. When the signal is under the realm of Poisson statistics one obtains at the relationship that

$$
\Delta \mathrm{I}_{\mathrm{M}}>2.33 * \sqrt{I_{M}}
$$

To assess sensitivity this deviation is traditionally compared to the characteristic signal $\Delta \mathrm{IM}_{\mathrm{M}} / \mathrm{IS}_{\mathrm{S}}$, here the total measured signal $\mathrm{I}_{\mathrm{M}}=\mathrm{I}_{\mathrm{S}}+\mathrm{I}_{\mathrm{B}}$ includes both characteristic peak ( $\left.\mathrm{I}_{\mathrm{S}}\right)$ and background ( $\left.\mathrm{I}_{\mathrm{B}}\right)$ components. In the limit when $\mathrm{I}_{\mathrm{B}}<<$ Is yields the relationship for Minimum Detectable Mass (MDM)

\section{$\operatorname{MDM} \propto \frac{2.33}{\sqrt{I_{S}}}$}

and when $\mathrm{IS}_{\mathrm{S}}<<\mathrm{I}_{\mathrm{B}}$ the Minimum Mass Fraction $(\mathrm{MMF})$

$$
\operatorname{MMF} \propto \frac{2.33}{\sqrt{I_{S} * \frac{I_{S}}{I_{B}}}}
$$

In practical studies it is seldom that either of these conditions ( $\mathrm{I}_{\mathrm{B}}<<$ IS or $\mathrm{IS}_{\mathrm{S}}<<\mathrm{IB}_{\mathrm{B}}$ ) are encountered since typically neither the characteristic nor background signals are insignificant with respect to each other. Further complicating this matter is the fact that both vary with specimen thickness. As such, when comparing simultaneously measured but different spectroscopies, it is important to scale both signals appropriately and, in this way, allowing one to make a relatively unbiassed assessment of the merits of 
one measurement versus the other. To do so, we have used the measurable quantity of $t / \lambda$, as a thickness normalization parameter. Using this a normalized spectral figure of merit (FoM) can be defined by scaling the respective intensities yielding:

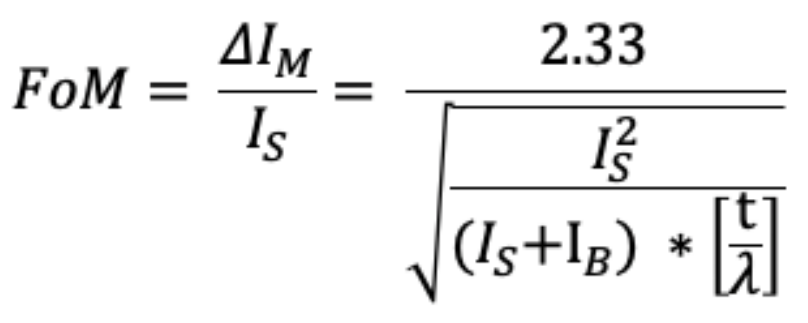

In the respective limits of: $\mathrm{IB}_{\mathrm{B}}<<$ Is or IS $<<\mathrm{IB}_{\mathrm{B}}$, this normalized FoM reduces to scaled values of MDM and MMF respectively. In both cases the smaller the value of FoM, then the higher the sensitivity, when respective measurements are made with different beam currents, the intensities should be similarly normalized.

To test the veracity of this formulation, we have compared the application of the FoM equation to XEDS and EELS data acquired from the same region of an ion milled single crystal specimen of NiO. These spectral results were all measured simultaneously under identical non-channeling conditions of thickness, beam current, time, and accelerating voltage. The data was acquired on a monochromated, image and probe corrected ThermoFisher/FEI Themis 60-300 microscope equipped with XFEG, SuperX EDS detector system, and GATAN Quantum ERS 965 GIF. The detailed results shown here were obtained at $60 \mathrm{Kev}$, however corresponding results at higher beam energies have also been obtained.

In Figure 1, we plot the spectra from the $\mathrm{NiO}$ specimen at three different relative thicknesses $(\mathrm{t} / \lambda \sim 0.3$, 1.1, and 2.0) here the Oxygen $\mathrm{K}$ and the Nickel L data are shown in the respective EELS (Fig 1A) and XEDS (Fig 1B) data. As expected both the signal and background increase with $t / \lambda$. This data is replotted in Figure 1C \& 1D, however, here the respective spectra are normalized to the background just prior to the OK signal. This scaling dramatically illustrates the change in the characteristic signal/background $\left(I_{S} / I_{B}\right)$ which occurs in the spectral data as a function of thickness. These results are similar to the $I_{S} / I_{B}$ measurements as a function of $\mathrm{t} / \lambda$ done $\sim 10$ years ago [1], however, they now reflect the advancements made in the respective spectrometer technologies which have significantly improved capabilities for both XEDS and EELS

The corresponding numerical integration of the spectra are presented in Figure 2. Here the EELS data was integrated over a $50-\mathrm{eV}$ window starting at each edge onset, while the XEDS signal was integrated over the full Gaussian peaks. Conventional background fitting power law formulations used in EELS and XEDS were used over the respective integration windows. In Figure 2A \& 2B, one can see that, over this thickness range, the signal/background in EELS changes by factors of as much as $350 \%$, while the XEDS varies by $<15 \%$. i.e. XEDS signal/background is relatively insensitive to thickness. This is not surprising given that the background in EELS is derived from multiple inelastic scattering events and is highly non-linear, while in contrast, the background in XEDS varies to a first approximation linearly with thickness as does its characteristic signal. In Figure $2 \mathrm{C} \& 2 \mathrm{D}$, we next compare the calculated FoM for the same spectral data, while identical trends with thickness are apparent. The FoM formulation does not mitigate the fact that the EELS signal still varies by $\sim 350 \%$ compared to $\sim 15 \%$ for XEDS. It continues 
to reinforce that for the current experimental systems that EELS is more sensitive than the XEDS system (i.e. the FoMEELS < FoMXEDS). However, it does very succinctly show that XEDS is less variable as a function of thickness than EELS.

The upcoming generation of x-ray detectors with increasing solid angles ( $\sim 2 \mathrm{sR})$ will, in the not to distant future, impact this difference as the collection efficiency and thus the raw XEDS signal has the potential to improve by factors of $\sim 3$. This will in some experimental situations lend the advantage to XEDS over EELS when only elemental spectroscopy is warranted [3].
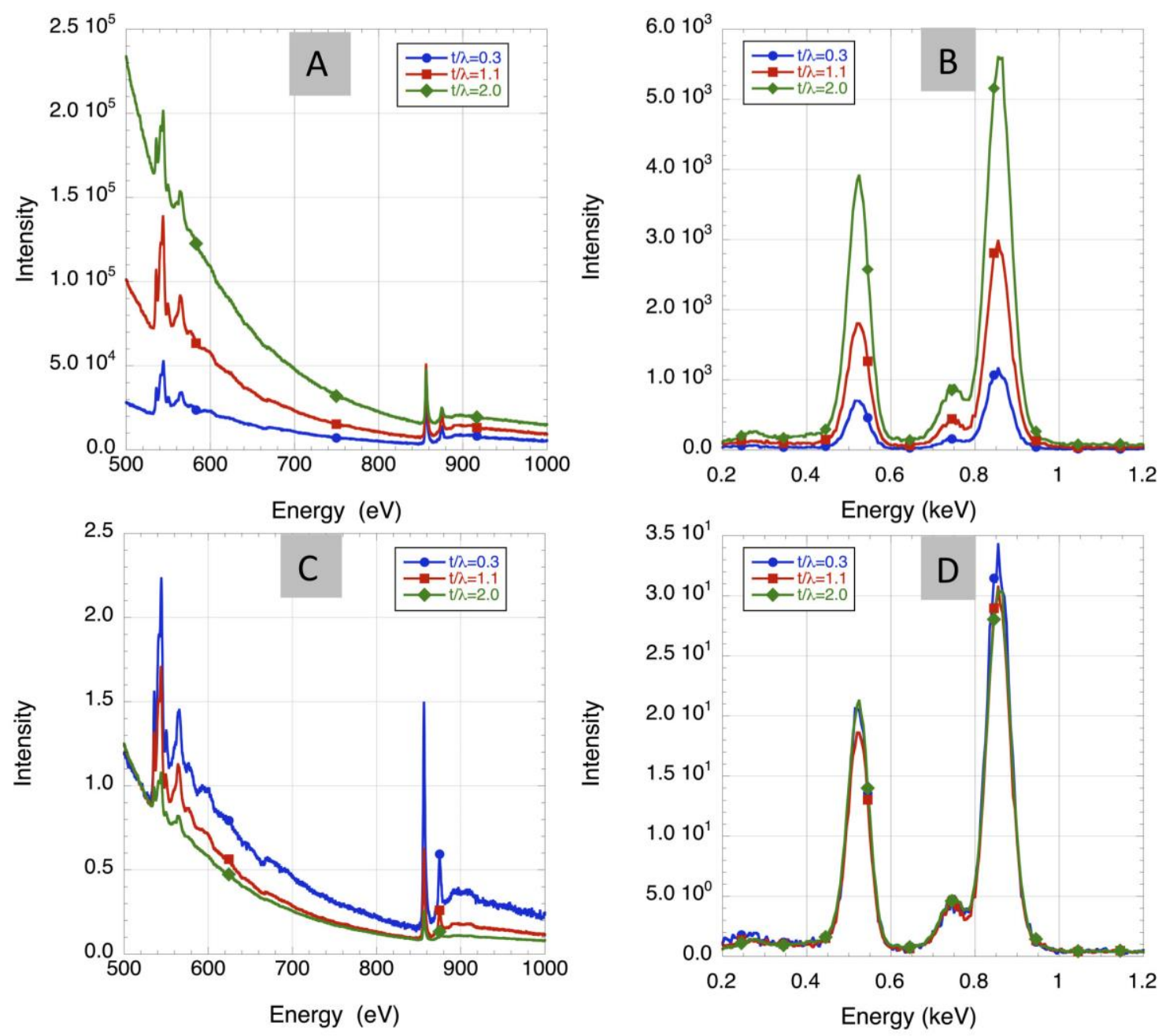

Figure 1. Unprocessed EELS (A) and XEDS (B) spectroscopic data from NiO specimen at $\mathrm{t} / \boldsymbol{\lambda}=0.3,1.1$, and 2.0. Background normalized spectral data EELS (C) and XEDS (D) to illustrate the change in Signal/Background (IS/IB) as a function of thickness, normalization to pre-OK background. 

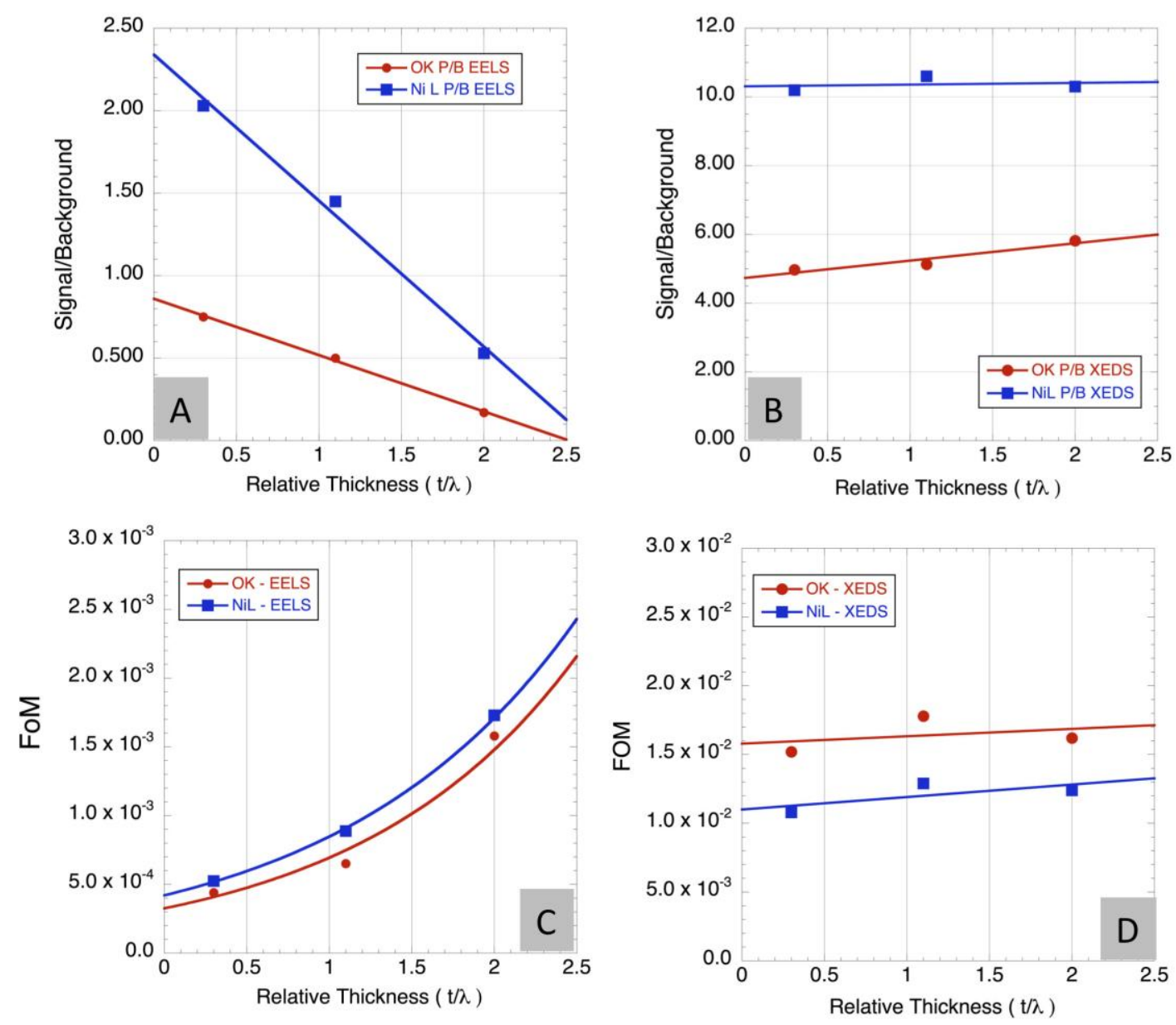

Figure 2. Signal to Background computed from spectra of figure 1 for EELS (A) and XEDS (B) illustrating the change in Signal/Background (IS/IB) as a function of thickness. Figure of Merit plot of EELS (C) and XEDS (D) results. Note the relatively small variation in FoM for XEDS compared to EELS vs $\mathrm{t} / \lambda$.

\section{References}

[1] Fraser H, Klenov D., Wang Y., Cheng H. Zaluzec N.J, Microsc. Microanal. 17 (Suppl. 2), 2011 590591, doi:10.1017/S1431927611003825

[2] Zaluzec N.J., Ultramicroscopy 203 (2019) 163-169 https://doi.org/10.1016/j.ultramic.2018.11.008

[3] This research was supported in part by Laboratory Directed Research and Development (LDRD) funding No. 2017-153-NO and the Photon Science Division at Argonne National Laboratory of the U.S. Department of Energy, Office of Science, Office of Basic Energy Sciences under contract No. DE-AC0206CH11357.The authors acknowledge the Sydney Microscopy and Microanalysis facilities at The University of Sydney. 